\title{
UV-Manipulation of Order and Macroscopic Shape in Nematic Elastomers
}

\author{
P.M. Hogan, A.R. Tajbakhsh, and E.M. Terentjev \\ Cavendish Laboratory, University of Cambridge, Madingley Road, Cambridge, CB3 OHE, U.K.
}

(Dated: October 22, 2018)

\begin{abstract}
A range of monodomain nematic liquid crystal elastomers containing differing proportions of photo-isomerisable mesogenic moieties, which turn from a rod-like to a kinked shape upon ultraviolet (UV) irradiation, was studied. Depending on the proportion and positional role of the photosensitive groups in the crosslinked polymer network, different types and magnitudes of response were found. The principle consequence of such photo-isomerisation is the destabilisation of the nematic phase, whose order parameter depends on temperature in a near-critical fashion. Accordingly, the effect of UV-irradiation is dramatically enhanced near the critical temperature, with the associated reduction in the nematic order parameter manifesting as a change in the macroscopic shape of the elastomer samples, producing a large uniaxial contraction. Theoretical analysis of this phenomenon gives a good quantitative agreement with experiment.
\end{abstract}

PACS numbers: $61.30 .-\mathrm{v}, 82.50 . \mathrm{Hp}, 61.41 .+\mathrm{e}, 82.30 . \mathrm{Qt}$

\section{INTRODUCTION}

The unique behaviour of liquid crystal elastomers derives from the intimate relationship between the elastic nature of the polymer network, and the ordering of their mesogenic, liquid crystalline moieties. Many interesting and unusual properties of these materials have been identified, and are summarised in recent review articles [1, 2, 3, 4, 5].

The property that is most relevant to this paper is the response of a liquid crystal elastomers macroscopic shape to temperature. A permanently, uniaxially aligned monodomain nematic liquid crystal elastomer network [6] will exhibit a spontaneous contraction along its director axis when heated towards its nematic-isotropic phase transition temperature [7, 8]. This phenomenon is due to the coupling between the average polymer chain anisotropy, and the nematic order parameter $\mathrm{Q}(\mathrm{T})$ [5]. The large variation in magnitude of resulting spontaneous uniaxial deformation depends strongly on the extent of this coupling between the elastic and the liquid-crystal degrees of freedom in the material [9, 10].

Accordingly, a mechanical action can be produced in response to a control signal which defines the underlying order parameter of the elastomer. Substantial macroscopic movement and shape changes are brought about by subtle variations in microscopic ordering. There are many methods of achieving mechanical motion in response to a control signal - or actuation - for many different applications [11], ranging from precise piezoelectric positioning devices to artificial muscles 112, 13. Liquid crystal actuators predominantly rely on thermal [8, 10, 13] and, to a much lesser extent, electromagnetic 114 control signals. Recently, however, a less invasive method of manipulating the underlying nematic order parameter in an elastomer network has been reported for the first time 15$]$.

When exposed to ultraviolet (UV) radiation, liquid crystal molecules containing photo-isomerisable groups, such as azobenzene, experience a reduction in their ne-

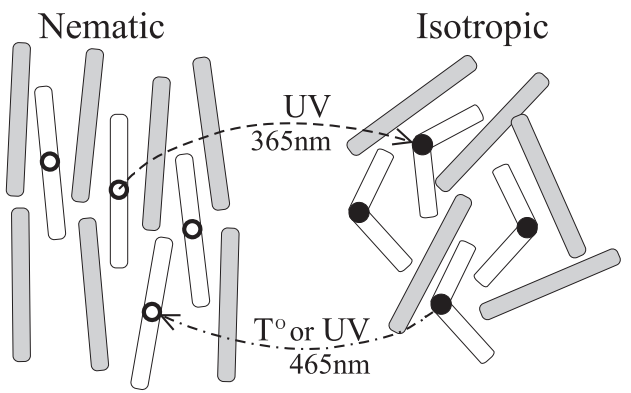

FIG. 1: Schematic depiction of nematic-isotropic phase transformation in a liquid crystal containing photo-isomerisable mesogenic molecules, which turn from a rod-like trans to a kinked cis conformation under the UV irradiation.

matic order and can therefore be induced to undergo a phase transformation from the nematic to the isotropic state [16, 17, 18]. This is due to the UV-induced cistrans isomerisation of the azo- $(\mathrm{N}=\mathrm{N})$ bond, whereby the photo-sensitive mesogenic molecules change from a rod-like shape, to a strongly kinked shape upon irradiation at a resonant wavelength of $365 \mathrm{~nm}[15$, 17, 19]. The rod-like shape serves to stabilise the liquid crystal phase, whereas the kinked shape has the opposite effect, acting as an impurity and destabilising the nematic phase, reducing its order parameter [17], Fig. 1. The dynamics of this process, and the reverse trans $\rightarrow$ cis reaction occurring on heating or irradiation at $465 \mathrm{~nm}$, are well studied in ordinary low-molar weight liquid crystals [17, 20].

With this method of non-invasive control over the degree of nematic ordering, there is now a new mechanism for actuation in liquid crystal elastomers. Such mechanical photo-induced actuation in nematic elastomers was first reported by Finkelmann et al. [15]. The degree of wide-spread, far-reaching interest in this new photomechanical effect is highlighted by recent popular articles in the New Scientist and The Financial Times [21, which appear even before any in-depth research had been conducted, and suggest exotic applications such as surgical 
micro-actuators.

In this article, a range of such photo-sensitive nematic liquid crystal elastomers is studied. Before investigating their varied response to ultraviolet light, their thermal responses are first characterised in section III A. The differing UV responses for the different sample compositions are presented in section III B. Section IV then focuses on the detailed quantitative analysis of the UV-mechanical response, comparing the results with theoretical expressions derived in section IV B.

\section{EXPERIMENTAL}

All starting materials and resultant aligned, monodomain nematic liquid crystal elastomers were prepared in the Cavendish Laboratory. The procedure for forming the side-chain polysiloxanes by the hydrosilation of the terminal vinyl group in the mesogenic moiety with the $\mathrm{Si}-\mathrm{H}$ bond of the polysiloxane chain, as well as the twostep crosslinking technique with a uniaxial stress applied after the first stage of crosslinking to produce and freeze the monodomain nematic alignment, has been developed over the years by Finkelmann et al. [6, 8, 22]. A number of minor modifications were made to the procedure, which are described in 10. The azobenzene compounds were all synthesised according to standard literature techniques [23, 24, 25]. A summary of the different mesogenic moieties used is given in Fig. 2.

From the structure of the molecular moieties one can deduce that all the side-group compounds are mesogenic rod-like units. PBB and BMAzo have a four carbon atom spacer, which induces a parallel orientation of the rods to the siloxane backbone resulting in a prolate chain anisotropy in the nematic phase, with its principal radii of gyration $R_{\|}>R_{\perp}$. The third rod-like side group, UBAzo, has a flexible spacer of eleven carbon atoms and is thus only expected to be weakly coupled to the siloxane backbone. There are three distinct types of di-vinyl crosslinking agents: the flexible, non-mesogenic DiUB, the rod-like DiUAzo and the main-chain nematic polymer DiMC, which contains a total of approximately 75 rod-like monomer units between its reacting vinyl groups. The polymer backbone onto which all these compounds were grafted was polyhydromethylsiloxane (PHMS), containing approximately $60 \mathrm{SiH}$ groups per chain, obtained from ACROS Chemicals.

The polymer networks were all crosslinked via the same hydrosilation reaction, in the presence of a commercial platinum catalyst COD, obtained from Wacker Chemie. The crosslinking density was $10 \mathrm{~mol} \%$ (molar percent, relative to the total number of $\mathrm{SiH}$ units) in all resultant elastomers.

Phase sequences were established on a Perkin Elmer Pyris 1 differential scanning calorimeter, which correlated with the critical temperatures obtained by the ther- mal expansion measurements (section III A). In all resulting elastomeric materials a broad nematic liquid crystalline phase below a certain critical temperature was observed, with optical microscopy and mechanical testing confirming that this was indeed the nematic phase. Some of the materials also possessed a second ordered phase at low temperatures, exhibiting high rigidity and little response to temperature or UV-irradiation, just above the glass transition. This additional phase was not a focus of the present work - all measurements were performed in the soft rubbery nematic phase of the elastomer samples.

Elastomer mechanical expansion measurements were obtained by freely suspending the samples in an active, glass-fronted Gallenkamp oven and measuring their variation in length with a travelling microscope. Cooling and heating rates were chosen such that thermal hysteresis effects were negligible (around $0.5^{\circ} \mathrm{C} / \mathrm{min}$, except for the sample containing DiMC, which required a much slower rate).

Samples (of approximate dimensions $30 \times 5 \times 0.4 \mathrm{~mm}$ ) were uniformly irradiated with a Merck VL-4.L UV lamp, providing a narrow band at $365 \mathrm{~nm}$ with an output of approximately $4 \mathrm{~W}$, at a distance of approximately $15 \mathrm{~cm}$. The temperature was determined with a thermocouple placed directly behind the samples.

\section{COMPARISON OF MATERIAL PROPERTIES}

A wide range of elastomer compositions and topologies (summarised in the Table I) was investigated, in order to probe different aspects of nematic elastomer response to UV-irradiation. It was universally found that the main consequence of UV-irradiation was the cis-trans isomerisation of the azobenzene groups, with a very low probability of side reactions. However, the differing compositions and topologies of the samples resulted in a range of substantially different magnitudes of the effect. One key factor to consider is the overall concentration of isomerisable azobenzene groups, which varied between 9 and $100 \mathrm{~mol} \%$ in our materials. Another crucial factor is the topological role of the crosslinker (cf. Fig. 2). The small, flexible crosslinking polymer, DiUB is deemed to have a minor effect on the overall mesogenic properties of the liquid crystal elastomer. This is quite unlike the other two crosslinkers - DiMC is a highly anisotropic main-chain nematic polymer containing $\sim 75$ rod-like monomer units, which can align itself and fold into hairpin defects in the nematic phase. DiUAzo contains a single, rod-like photoisomerisable unit of its own; its effect on the mechanical response of the elastomer network to UV-irradiation was the subject of the pioneering study by Finkelmann et al. [15. 


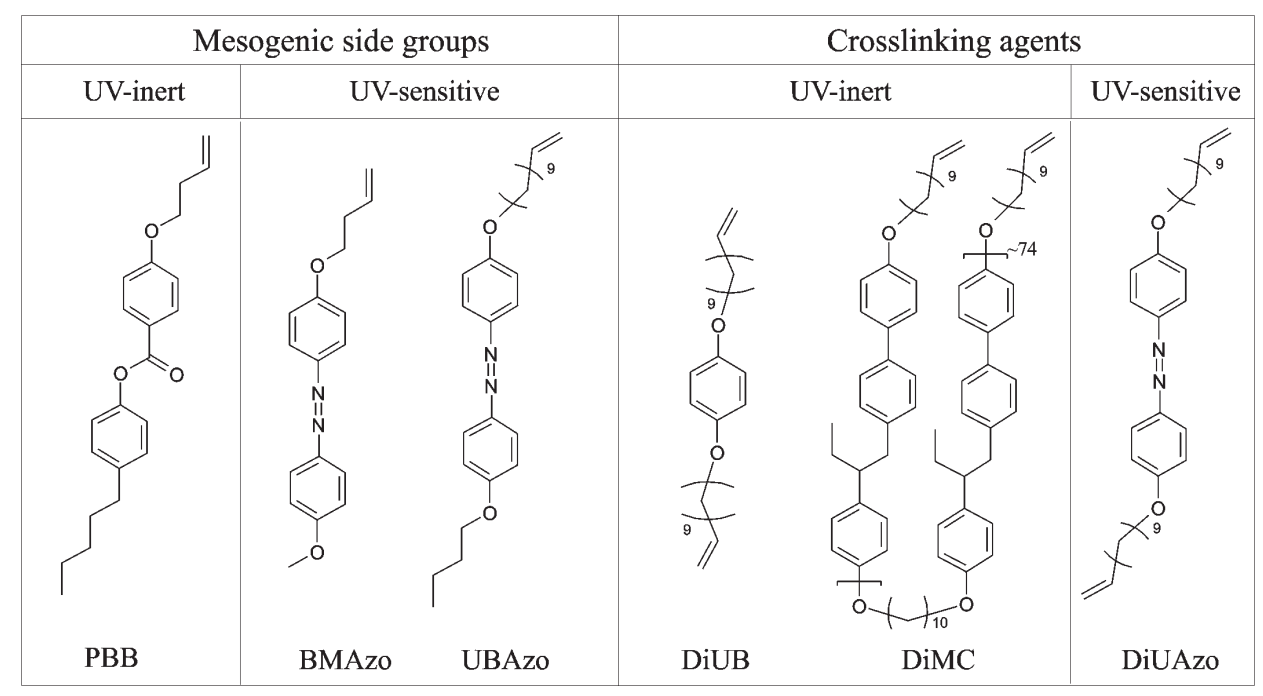

FIG. 2: The mesogenic moieties used are abbreviated as follows: PBB - 4-pentylphenyl-4'-(4-buteneoxy)benzoate; BMAzo - [4-(4-buteneoxy)-4'-methyloxy]azobenzene; UBAzo - [4-(11-undeceneoxy)-4'-butyloxy]azobenzene; DiUB - di1,4-(11-undeceneoxy)benzene; DiMC - $\alpha$-4-[1-(4'-11-undeceneoxybiphenyl)-2-phenyl]butyl- $\omega$-(11-undeceneoxy) poly-[1-(4oxydecamethyleneoxy)-biphenyl-2-phenyl]butyl ( 75 units long); DiUAzo - di-[4-(11-undeceneoxy)]azobenzene.

TABLE I: Compositions, and corresponding abbreviations, of the different photo-sensitive nematic elastomers studied in this work. The composition is presented in two forms: first, in mol\% of corresponding moieties; second, the proportion of rod-like mesogenic units in the overall composition. The rod-like side groups are a combination of a "standard" nematic PBB and one of two kinds of the azo-containing groups (cf. Fig. 2), specified in the second column of the table. The crosslinking density in all cases is kept at a constant $10 \mathrm{~mol} \%$.

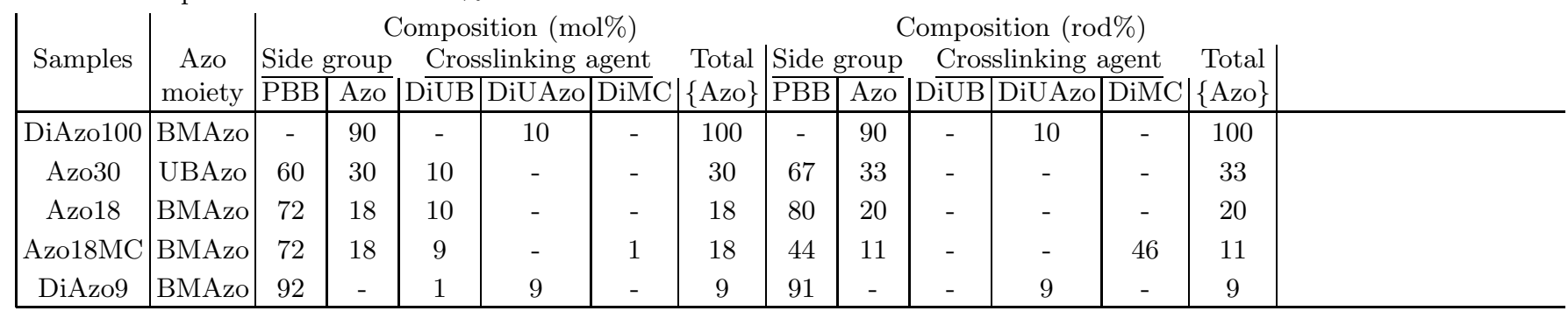

\section{A. Uniaxial thermal expansion}

Before their response to ultraviolet light was investigated, the spontaneous uniaxial thermal expansion of each of the samples was first characterised (Fig. 3). This determines the extent of the coupling between the local nematic order, described by the scalar order parameter $Q(T)$, and the average elastomer network anisotropy, measured as the spontaneous uniaxial extension $\lambda=$ $L / L_{0}$, where $L$ is the current length of the sample, and $L_{0}$ its length in the isotropic phase (the latter is always shorter for an elastomer with a prolate backbone configuration, as is the case for all our materials).

Empirically, one can relate the uniaxial thermal expansion of a liquid crystal elastomer, $\lambda$, to the underlying nematic order parameter $Q(T)$, which can be independently determined by either wide-angle X-ray scattering, or from the optical birefringence of the elastomer. It has been shown [9, 22] that the relationship between the macroscopic shape and the nematic order of liquid crystal elastomers can be very well described by a direct linear proportionality, $\lambda=1+\alpha Q$. The constant $\alpha$ is a sensitive function of the average network anisotropy, varying from $\alpha \sim 0.08$ in weakly anisotropic elastomers to $\alpha \sim 3$ or greater in materials containing main-chain crosslinkers [9]. Such a relationship between the spontaneous uniaxial extension and the underlying nematic order is wellunderstood theoretically within the molecular model of equilibrium nematic polymer networks [5].

However, a conceptual problem resides in the values of the nematic order parameter itself. The thermal expansion data sets for all our materials, as well as the analogous studies by other groups, e.g. [13, 22, were all found to follow $\lambda=1+\alpha Q$ very faithfully, but with $Q$ varying in the manner of a virtual second-order phase transition, $Q \propto\left|T-T_{\text {ni }}\right|^{\xi}$ 26]. In our present materials the critical exponent of the apparent critical behaviour, $\xi$, takes values between 0.14 and 0.31 (Table III). There are several possible reasons for this observation of an ap- 

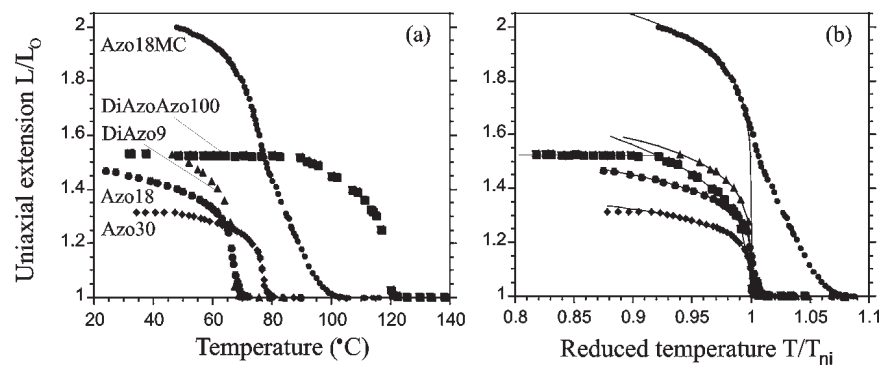

FIG. 3: Uniaxial thermal expansion of nematic elastomers. Plot (a) shows the raw data for the extension of the freely suspended elastomers. With plot (b), the temperature for each sample is scaled with the extrapolated nematic-isotropic transition temperature $T_{\text {ni }}$. Below this point, each data set is fitted with a critical power law of the form $(\lambda-1) \propto \mid T-$ $\left.T_{\mathrm{ni}}\right|^{\xi}$. The main-chain containing sample Azo18MC shows a pronounced pre-transitional expansion (see text).

parent critical behaviour in a system that, by symmetry arguments, should undergo a discontinuous first order transition. One likely explanation is that the kinetic response of the rubber-elastic network is such that it is unable to perform the discontinuous jump required by the changing underlying nematic order parameter. Such an explanation, although appealingly simple, has several weaknesses, the main one being that practically no dependence on cooling/heating rate is observed, which should be expected if the kinetic retardation is at work.

Another possibility is that in forming the monodomain nematic materials, an effective aligning molecular field is frozen in by crosslinking. This makes the first-order nematic-isotropic transition supercritical and, essentially, eliminates the true isotropic phase altogether: even at high temperature there would still be a small residual order remaining. This could still be the case, even though in our materials we could not experimentally detect any residual order above $T_{\text {ni }}$ and observe a clear and welldefined heat signature of the transition on DSC.

A perhaps more intriguing possibility is the presence of quenched sources of random disorder in the nematic field (the network crosslinking points), which genuinely transforms the weak first order transition into a continuous one, an effect already discussed in the context of spin glasses [27]. The present paper does not attempt to address this issue. We focus instead on a different physical property of a photo-sensitive nematic elastomer and simply use this experimentally observed, continuous "critical" variation of $Q(T)$ as the foundation for describing the UV-induced response of such an elastomer.

It seems that a consistent value of the "critical exponent" is obtained, $\xi \sim 0.2$. The anomalously small value of $\xi$ for Azo18MC can be attributed to the significant difference in this sample's response time from all the others: kinetic effects are markedly relevant and the long DiMC chains have been shown to slow down, and even completely freeze their dynamics, when in the hairpin regime of their nematic conformation [28]. This would result
TABLE II: Experimentally determined transition points, $T_{\mathrm{ni}}$ (used as a parameter in fitting by the power-law), and the apparent critical exponents, $\xi$, for all materials studied. The samples are ranked by their overall azo-content.

\begin{tabular}{|c|c|c|} 
Sample & $T_{\mathrm{ni}}^{(0)}\left({ }^{\circ} \mathrm{C}\right)$ & $\xi$ \\
\hline DiAzo100 & 120.3 & 0.31 \\
Azo30 & 76.8 & 0.21 \\
Azo18 & 67 & 0.19 \\
Azo18MC & 75.2 & 0.14 \\
DiAzo9 & 66.5 & 0.20 \\
\hline
\end{tabular}

in the non-equilibrated thermal expansion of the sample lagging the actual, equilibrated order parameter. This sample also shows a very significant pre-transitional effect, with the residual nematic order and apparent sample extension persisting far above the critical temperature. This phenomenon has been examined in a separate study [10] and is due to a composite nature of this material in which the side-chain and main-chain polymer chains are, crudely, in equal proportion. Another point of interest is the presence of highly ordered phases in DiAzo100 and Azo30 at lower temperatures, where the dependence of $Q$, and hence $\lambda$, on temperature deviates from the attributed nematic behaviour, and becomes essentially constant. However, this will not be dwelt upon, as this work focuses exclusively on the nematic phase and the nematic-isotropic phase transition.

The observed dependence of $Q$ and $\lambda$ on temperature is very important in analysing and modelling the response of the liquid crystal elastomers to UV-irradiation. In doing so, one sample (Azo18), which proved to have the most pronounced response to UV-irradiation, is studied in greater detail in Section IV.

\section{B. Response to UV-irradiation}

In order to compare different compositions and crosslinking topologies of the nematic elastomers in their response to UV-irradiation, all samples were equilibrated before irradiation at approximately $10^{\circ} \mathrm{C}$ below their critical temperature $T_{\text {ni }}$, determined by the thermal analysis in section III A. Such proximity to $T_{\text {ni }}$ also aims to maximise the variation of $\lambda$, whilst avoiding the singularity at the phase transition.

During irradiation, the sample temperature was, without exception, found to increase in an approximately exponential manner, mirroring the behaviour of the spontaneous uniaxial extension, $\lambda$. Similarly, as the elastomers relaxed after the radiation was switched off, the measured temperature again mirrored $\lambda$, decreasing back to its pre-irradiated value. In both cases, this exponential variation in temperature was found to be modulated by a small-amplitude oscillatory behaviour. Possible explanations for this thermal phenomenon involve a trivial heat- 


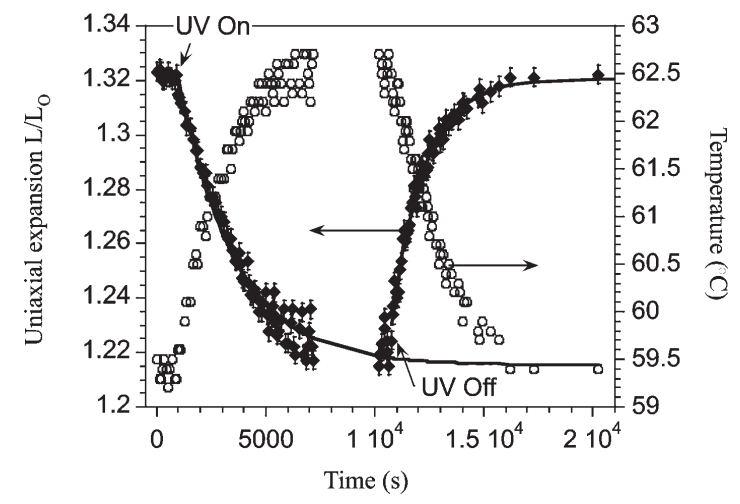

FIG. 4: The response of Azo18 to UV irradiation. Both the relative length of the sample (uniaxial strain $\lambda$, measured with respect to the isotropic state: filled symbols) and the measured temperature (open circles) are shown. The $\pm 0.1^{\circ} \mathrm{C}$ errors in temperature have been omitted for clarity. Solid lines are fits of both the on and off branches by a simple exponential, which at this point should be regarded as only a guide to the eye.

ing effect from the UV lamp interfering with the active temperature control of the oven. However, such an additional heating power should not result in the overall rise of saturated temperature value, as seen in Fig. 4: the oven would be expected to reduce its own heating and bring the temperature back to its set value. Also, it would be an inconceivable coincidence if the characteristic rate of the oven heating system are exactly the same as that of all our different materials.

By closely examining the Fig. 1 it becomes clear that we observe the effect of spontaneous sample heating, occurring due to the exothermic nature of isomerisation of azobenzene groups. In a following section we shall describe the population dynamics between the trans and cis $\mathrm{N}=\mathrm{N}$ isomers. The relative proportion of these states is controlled by the rates of forward and reverse reactions, which reach the balance in the saturated state (after a long time of irradiation). If one assumes that the heat release on the return to the trans ground state is proportional to the rate of change of cis concentration, $\dot{n}_{c i s}$, then the observation in Fig. 1 becomes sensible. Initially, all azobenzene groups are in their trans state and the sample is thermally equilibrated with the oven (at $59.5^{\circ} \mathrm{C}$ in this case). On irradiation, the increasing number of cis isomers is created, and also accompanied by an ever increasing relaxation flux back to the trans state. This heats the sample, at the rate exactly matching the rate of isomerisation itself. Finally, a saturation state is reached, when the UV-stimulated trans $\rightarrow$ cis flux is equal to the relaxation flux: at this stage the heat release in the sample is constant and maximal. On switching the UV-light off the exothermic cis $\rightarrow$ trans relaxation continues to heat the sample, but with a decreasing power as the overall concentration $n_{\text {cis }}$ decreases.

All the samples responded to UV-irradiation in a manner similar to Azo18, illustrated in Fig. 因, differing only in
TABLE III: Sample contractions upon UV-irradiation, relative to the expected thermal contraction due to the accompanied temperature increase above the starting point (last column). The increase of this ration above unity is a genuine measure of the effect of UV isomerisation.

\begin{tabular}{|c|c|c|} 
Sample & $\Delta \lambda / \Delta \lambda(T)$ & $T_{\exp }\left({ }^{\circ} \mathrm{C}\right)$ \\
\hline DiAzo100 & 1.21 & 110.5 \\
Azo30 & 1.49 & 66.9 \\
Azo18 & 2.82 & 57 \\
Azo18MC & 1.03 & 65.0 \\
DiAzo9 & 1.63 & 56.8 \\
\hline
\end{tabular}

the magnitude and speed of their response. The resultant contraction upon irradiation for each sample, relative to the expected contraction due to the aforementioned increase in temperature, are summarised in Table III. As can be seen, the proportion of UV-isomerisable azobenzene rods in the sample is by no means a direct indication of its response to UV-irradiation, interpreted as the maximal sample extension achieved on irradiation.

Azo18 gave the largest response to radiation, but the very similar Azo30, with its higher proportion of azo-rods, gave a significantly smaller response. This must be due to the position of the azo-group within the corresponding mesogenic moiety - with Azo30, it is only weakly coupled to the polymer backbone by a long spacer, in contrast to Azo18, where a strong parallel alignment between the rod and the backbone is induced. This could result in the kinked cis-form of the moiety having less of a destabilising effect on the backbone anisotropy, and so the elastomer response to UVirradiation is curtailed.

DiAzo9 is very similar to the material studied by Finkelmann et al. 15. Despite containing less than a third of the proportion of azobenzene rods found in Azo30, its response to UV-irradiation is comparable in magnitude, which should be attributed to the crucial position of the photo-isomerisable group, in the centre of the predominant, short crosslinker.

Azo18MC might have been expected to have a very large response compared to the other materials due to its large backbone anisotropy and large coupling constant to the underlying order parameter, illustrated by its large thermal expansion amplitude. However, this was definitely not the case for UV irradiation. One may speculate the cause may be not only that the actual proportion of azobenzene rods was quite low for this sample (much of the volume is occupied by the main-chain polymer crosslinker), resulting in a smaller absolute change in the order parameter, but more importantly, that large DiMC chains can essentially freeze the elastomer dynamics in response to changes in $Q$ [28], quenching its manifestation through $\lambda$.

All the mesogenic groups and crosslinkers in DiAzo100 were photo-sensitive, and so one might have expected 
an exceptional mechanical response to UV-irradiation. However, the critical temperature, $T_{\text {ni }}$, of this sample was much higher than that of the other materials, and to maintain consistency between experiments on different samples this required an initial equilibrium temperature of approximately $110^{\circ} \mathrm{C}$. At such a temperature the thermally-activated relaxation taking the azobenzene groups from their metastable kinked cis-shape back to the rod-like trans-shape would be much accelerated. As a result, one is perhaps unable to isomerise a significant proportion of azobenzene rods at any given moment of time. A lower equilibrium population of cis-isomers would then result in a smaller overall change in $Q$, and hence in $\lambda$.

The solid curves in Fig. 1 are simple exponential fits to the data, for both the UV-on and the UV-off states. Apparently, it is a reasonably good fit. However, the parameters are rather arbitrary and appear to be quite unrelated to the corresponding spontaneous thermal expansion curves. It will shortly be seen that a different, universal theoretical dependence on temperature and irradiation time describes the data with a much greater degree of fidelity. Accordingly, the solid lines in Fig. serve only as a rough guide to the eye.

\section{MANIPULATION OF NEMATIC ORDER}

\section{A. Population dynamics}

The internal energy of rod-like trans, and the kinked cis mesogenic moieties is adequately described as a twolevel potential well, with the former configuration energetically more stable than the latter (Fig. 5). In the experiments conducted, there are three possible ways of moving between states, each characterised by a separate time constant:

- Thermal excitations up, from trans to cis, with the characteristic time given by $\tau_{t c}=\tau_{0} e^{U / k T}$, with $\tau_{0}$ the bare attempt rate.

- Thermally driven relaxation down, from cis to trans, with the time constant $\tau_{c t}=\tau_{0} e^{\Delta / k T}$.

- UV-isomerisation, occurring with the rate $1 / \tau_{\mathrm{uv}} \equiv \eta \propto$ radiation intensity.

One expects that the barrier height $U$ is much greater than the metastable state depth $\Delta$, and hence one can neglect the rate of spontaneous, thermally driven trans to cis transitions, although certainly not its reverse.

Let us assign the total (fixed) number density of photoisomerisable rod-like groups in a given elastomer as $n_{0}=$ $n_{\text {trans }}+n_{\text {cis }}$, with the only independent variable defined as $n \equiv n_{\text {trans }}$, so that $n_{\text {cis }}=n_{0}-n$. Thus, the rate of change in the population of trans isomers can simply be written as;

$$
\frac{\partial}{\partial t} n(t, T)=-\eta n-\frac{1}{\tau_{t c}} n+\frac{1}{\tau_{c t}}\left(n_{0}-n\right)
$$

In the above approximation, the relaxation time $\tau_{t c}$ is as-

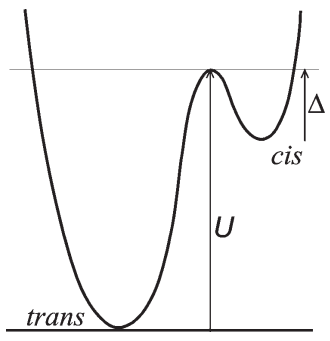

FIG. 5: Schematic depiction of the potential energy profile of a photo-sensitive moiety verses configuration, showing the relative stabilities of the cis and trans isomers.

sumed to be very large, and can therefore be neglected. The appropriate initial condition is then $n_{t=0}=n_{0}$ (that is, all azobenzene groups are in their rod-like trans conformation) and the dynamic solution to Eq. 1 is simply;

$$
\begin{gathered}
n(t, T)=\frac{n_{0}}{1+\tau_{c t} \eta}\left[1+\tau_{c t} \eta \exp \left(-t / \tau_{\text {eff }}\right)\right] \\
\text { with } \tau_{\text {eff }}=\left(\eta+1 / \tau_{c t}\right)^{-1}
\end{gathered}
$$

Hence, one can see that the increasing concentration of kinked cis-isomers, which act as impurities for the nematic ordering, will eventually reach a plateau due to the opposing thermal back-reaction, itself characterised by the rate $1 / \tau_{c t}$. Both the rate and the final saturation value of the isomer density are crucially dependent on the irradiation rate $\eta$; this could be different for different materials, sample shapes and experimental conditions as it is affected, among other factors, by the radiation absorption in the medium.

The relaxation process, after switching the radiation off (that is, setting $\eta=0$ ), is the thermally driven flux of cis-isomers into the trans ground state over the barrier $\Delta$. Assuming a fully saturated irradiated state, with $n_{\text {cis }}=$ $n_{0}-n_{\infty}=\tau_{c t} \eta /\left(1+\tau_{c t} \eta\right)$ at $t=0$ (and still neglecting the spontaneous thermal trans $\rightarrow$ cis process), results in the relaxation law

$$
n(t, T)=n_{0}\left[1-\frac{\tau_{c t} \eta}{1+\tau_{c t} \eta} \exp \left(-t / \tau_{c t}\right)\right] .
$$

Naturally, the relaxation time scale is purely $\tau_{c t}$, a property of an individual azobenzene group, which should remain at least approximately universal between all different samples under study.

\section{B. Macroscopic shape and nematic order}

The influence of impurities on the nematic order is well studied [29]. One of the simplest ways of representing their effect is via the Landau-de Gennes mean-field formulation, which describes the local free energy density as a power series in the magnitude of the scalar order parameter, $Q$. Such an approach is commonly used in the thermodynamics of second order phase transitions, 


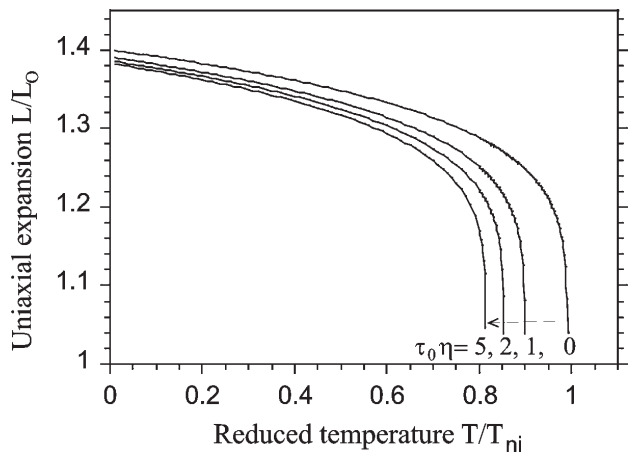

FIG. 6: The theoretically calculated effect of increasing the intensity of UV-irradiation (measured by the effective nondimensional rate $\tau_{0} \eta$ ) on the uniaxial thermal extension of a photo-sensitive liquid crystal elastomer, according to Eq.

as well as in application to liquid crystals. Although it is much less suitable for the nematic-isotropic transition in liquid crystals, which should be first-order by symmetry, many useful qualitative results are obtained within the Landau-de Gennes formalism.

However, in view of many limitations of such a phenomenological mean-field theory, we adopt a different approach. In section III A, the actual form of the dependence of the order parameter on temperature was established experimentally as being a virtual, continuous transition with the apparent critical exponent $\xi$ quite far from the mean-field value predicted by the Landaude Gennes approach (and certainly appears a continuous transition). Therefore, we simply take the empirical dependence, $Q \propto\left|T-T_{\mathrm{ni}}\right|^{\xi}$, and modify it by substituting in the impurity-shifted critical temperature, $T_{\mathrm{ni}}=T_{\mathrm{ni}}[n(t, T)]$.

The effect of impurities on the critical temperature is, in the first approximation, a linear function of the population of cis-isomers. The size of this population, $n_{\text {cis }}$, defined above as $n_{0}-n(t)$, is itself a function of time;

$$
T_{\mathrm{ni}}(n)=T_{\mathrm{c}}-\beta\left[n_{0}-n(t)\right]
$$

where $T_{\mathrm{c}} \equiv T_{\mathrm{ni}}^{(0)}$ is the transition temperature in the "pure" material containing only the trans rod-like units (given in the Table II for our materials). Combining this with the known dependence of the spontaneous uniaxial sample extension, $\lambda$, on $Q$, one obtains the following relation to describe the observed mechanical response to UV-irradiation

$$
\begin{aligned}
& \lambda_{\mathrm{uv}}(t, T)= \\
& =1+\alpha\left[T_{\mathrm{c}}-\beta n_{0}\left(1-\frac{\tau_{c t} \eta}{1+\tau_{c t} \eta}\left(1+e^{-t / \tau_{\mathrm{eff}}}\right)\right)-T\right]^{\xi}
\end{aligned}
$$

Plotting $\lambda$ against temperature for different values of the intensity parameter $\eta$, Fig. 6 , one sees that the expected consequence of UV-irradiation is simply to lower the critical temperature of the nematic-isotropic phase transition.

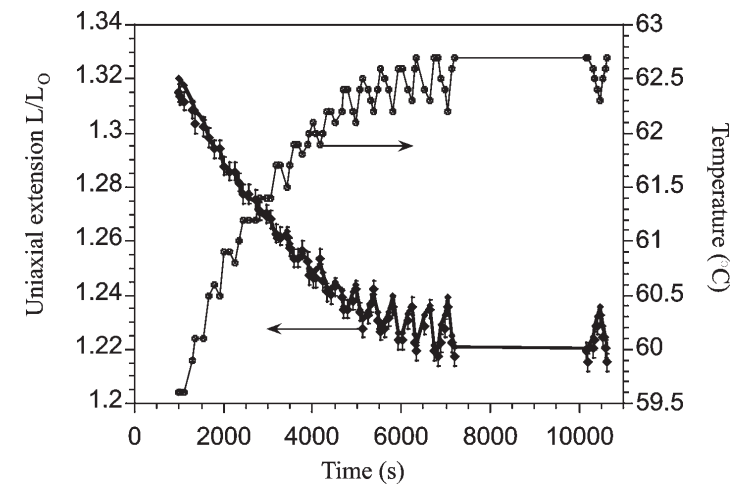

FIG. 7: The response of Azo18 to UV-irradiation, and the corresponding theoretical fit by Eq. (5), solid line. Note that even the minor intrinsic temperature oscillations are, in fact, directly manifested in $\lambda(t, T)$, and are almost perfectly described by the theoretical model (see text).

The relaxation of sample shape after switching the radiation off is determined by the rate of recovery of the initial population of trans isomers, according to Eq. (3);

$$
\lambda_{\text {off }}(t, T)=1+\alpha\left[T_{\mathrm{c}}-\beta n_{0} \frac{\tau_{c t} \eta}{1+\tau_{c t} \eta} e^{-t / \tau_{c t}}-T\right]^{\xi},
$$

where, as in the Eq. (3), we have assumed that a fully saturated state was achieved on preceding irradiation.

One important parameter has to be used in the further analysis: the relaxation time scale of the cis $\rightarrow$ trans pro-

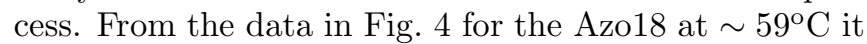
is estimated as $\tau_{c t} \approx 1479 \mathrm{~s}$, assuming that the dynamics is dominated by the rate of isomerisation (and not, for instance, the viscoelastic relaxation of a rubbery network). In the following analysis of experimental data, the effect of (Arrhenius) temperature variation of $\tau_{c t}$ within a given data set are, in the first approximation, neglected. This is justified by the relatively narrow range of absolute temperatures over which a given data set varies. However, across data sets, $\tau_{c t}$ is shown to vary significantly with temperature.

\section{Nematic destabilisation by UV radiation}

Applying the Eq. (5) to the data in Fig. (1, one obtains a remarkably improved fit (Fig. 1). In analysing the data we take the fixed values for the coefficient $\alpha=0.22$ and the exponent $\xi=0.19$, as determined by the thermal expansion measurements of section III A and the relaxation time $\tau_{c t}=1479 \mathrm{~s}$, as estimated from a crude exponential fit of Fig. 1 . The values of current temperature $T$ are taken from the corresponding experimental data points. The free parameters are, thus, only the radiation rate $\eta \Rightarrow 2.2 \cdot 10^{-4} \mathrm{~s}^{-1}$ and the product $\beta n_{0} \Rightarrow 11.8$, which is a measure of the overall concentration, and the efficiency, of azobenzene groups. 
The result of such a fit for $\lambda(t, T)$ is very satisfying. The initial quasi-linear contraction and its subsequent rapid flattening and saturation are fully described, unlike the rough exponential fit in Fig. 1 . More importantly, we now discover that what looked like noise in both the temperature and the strain $\lambda(t)$ in the initial graph, Fig. 1 , is in fact a consistent small-amplitude temperature oscillation (probably due to the active oven control operation), which is also fully reproduced in the mechanical shape of the sample. This is a rather stringent test of the theoretical model and the parameters used to fit the experimental data.

All the other data sets for Azo18 at different temperatures are fitted equally well, as shown in Fig. 8. Once again, we fix the value of strain-order coupling coefficient $\alpha=0.22$ and the exponent $\xi=0.19$, as determined by the thermal expansion measurement for this sample, and the relaxation time $\tau_{c t}=1479 \mathrm{~s}$. On physical grounds, we also insist that the coefficient $\beta n_{0}$ remains constant $(\sim 12)$ between these data sets, since it is determined by the intrinsic properties of the nematic order and the given concentration of azobenzene groups. With these stringent constraints, one obtains the values of the only remaining free parameter, the effective rate of induced isomerisation. The inverse of $\eta$, the characteristic time for the photo-induced trans $\rightarrow$ cis isomerisation, $\tau_{\text {uv }}=1 / \eta$, is plotted in Fig. 9 (a) and has an average value $\tau_{\mathrm{uv}} \sim 3 \cdot 10^{3} \mathrm{~s}$. Although there is a certain spread in these values, we find the outcome of this analysis quite satisfactory given the number of constraints imposed on the fitting, certain error in the data and, in spite of that, a clear evidence that not only the overall trend but also the small oscillations of temperature are well reproduced by the Eq. (5).

The values of the characteristic time for the reverse thermal cis $\rightarrow$ trans relaxation, $\tau_{c t}$, are consistent with those reported in the literature for azobenzene photochemistry [25], as well as within themselves, Fig. 9(b). The crude estimate of the cis $\rightarrow$ trans energy barrier from the Arrhenius fit of the relaxation time returns a reasonable value $\Delta \sim 4 \cdot 10^{-20} \mathrm{~J} \sim 10 k T$. This is in contrast with the isomerisation time $\tau_{\mathrm{uv}}$, which was found to be a constant, or very slowly decreasing function of temperature. However, the errors in determining $\eta$ were large, and there is much room for further studying its dependence on temperature.

\section{The vicinity of critical point}

Since the effect of UV radiation on a nematic rubber containing isomerisable azobenzene groups is clearly identified as an effective shift of critical temperature of the underlying nematic-isotropic phase transition, the question arises - what would be the response when the material is irradiated at a temperature close to the "bare" critical point $T_{\mathrm{c}}$ ? In this case, the increasing concentration of cis-impurities should take the system past the transition line into the isotropic phase. Continuing the study of Azo18, we now subject it to UV-radiation at a temperature $T_{\exp } \approx 63^{\circ} \mathrm{C}$, only $4^{\circ}$ below $T_{\mathrm{c}}$.

Fig. 10 indicates that the mechanical response of the sample on irradiation, $\lambda(t)$, starts exactly in the same way as at all other temperatures. However, the initial steep drop in $\lambda$ (and in the underlying nematic order $Q$ ) rapidly reaches the point of $\lambda=1$ (or $Q=0$ ); the material becomes fully isotropic and no longer has any macroscopic response to the further increase of the population of cis-isomers. The irradiation continues for some time, as illustrated by the continuing rise in temperature, which nevertheless has never reached the "bare" $T_{\mathrm{c}}$. Then the UV-light is switched off and the temperature begins to fall, in the same way as all previous measurements have shown. However, nematic order is not established for some time: clearly the concentration of cis-impurities has to decrease sufficiently to allow the nematic phase to appear at a given temperature.

Accordingly, there are two simultaneous thermal effects in the material. One is the rise (in the UV-on state), or fall (in the off state), of the actual temperature of the sample. The other is the underlying shift of the critical temperature of the nematic phase transition, described by the Eq. (4) with the time-dependent concentration $n(t)$ given by Eqs. (2) and (3), respectively (in the latter case of thermal cis $\rightarrow$ trans relaxation, Eqs. (3) and (6) assume that the state of full saturation has been reached on irradiation, otherwise a different initial condition has to be used, altering the prefactor to the exponential). It is straightforward to apply the corresponding analysis and determine the time when, in the UV-on state, the decreasing critical temperature $T_{\mathrm{ni}}=T_{\mathrm{c}}-\beta\left[n_{0}-n(t)\right]$ becomes equal to the increasing sample temperature. Equally, in the UV-off state, we find the time needed for the increasing $T_{\mathrm{ni}}$ to intersect the decreasing actual temperature $T$.

Applying this analysis to Azo18 in Fig. 10, one obtains that the nematic order disappears at $t \approx 1100 \mathrm{~s}$ and subsequently re-enters the nematic phase, is $t \approx 4850 \mathrm{~s}$. Both times are marked with arrows in Fig. 10: clearly the agreement with the observed change in the sample length, $\lambda=L / L_{0}$, is excellent. This is another confirmation of the theoretical model, which assumes simple thermal activation laws for characteristic times, the linear dependence of critical temperature on the concentration of cis-isomers and, much less trivially, the empirical critical dependence of the nematic order parameter $Q \propto\left|T-T^{*}\right|^{\xi}$.

\section{CONCLUSIONS}

The derivation of Eq. (5) in section IVB, cumulating in the analysis of relaxation data sets in Figs. 겨, makes it clear that, once the number of kinked moieties reaches a steady-state population, the resultant effect of UV-irradiation is equivalent to simply reducing the criti- 


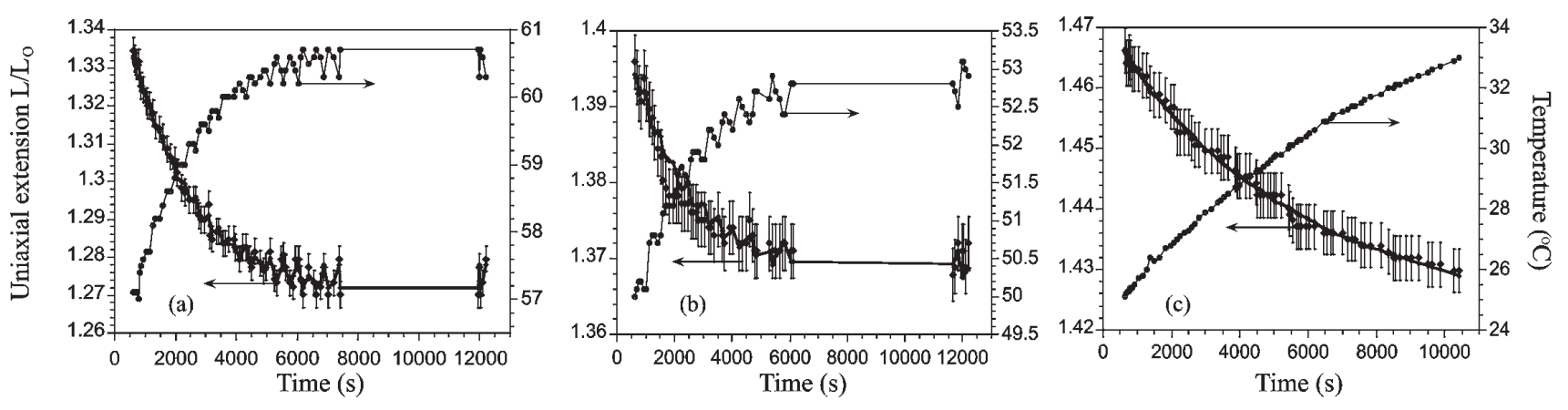

FIG. 8: The response of Azo18 to UV-irradiation at different starting temperatures, $\sim 57,50$ and $25^{\circ} \mathrm{C}$ for plots (a), (b) and (c), respectively. The corresponding description of $\lambda(t, T)$ with the theoretical model, Eq. (5), is shown by the solid lines. The relative error increases for lower temperatures due to the smaller overall magnitude of the UV-response.
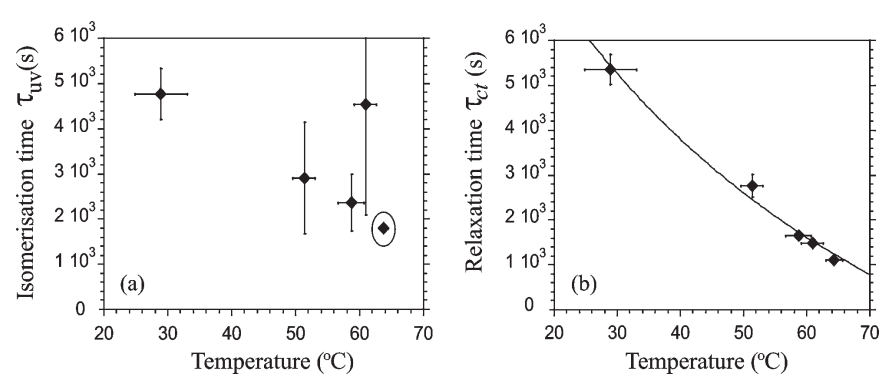

FIG. 9: (a) The irradiation time constants, $1 / \eta$, at different temperatures, are all of the same order of magnitude and show a constant or slowly decreasing dependence on temperature. The circled point, near $T_{\mathrm{ni}}$, has an ambiguously high error associated with it (see section IVD). (b) Relaxation times $\tau_{c t}$ gleaned from a simple exponential fit of the relaxation data. The activation temperature dependence allows a crude estimate of the isomerisation energy barrier $\Delta \sim 4 \cdot 10^{-20} \mathrm{~J} \sim$ $10 k T$.

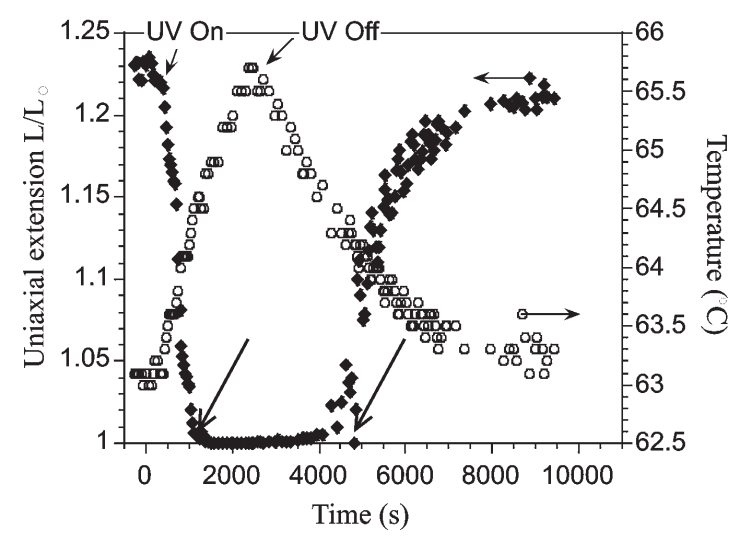

FIG. 10: The response of Azo18 to UV-irradiation in the vicinity of critical point. Arrows indicate the moments of time when the material first becomes fully isotropic on irradiation, and then re-enters the nematic phase on relaxation (the associated measured temperature of $\sim 64^{\circ} \mathrm{C}$ is clearly insufficient to account for this transition).

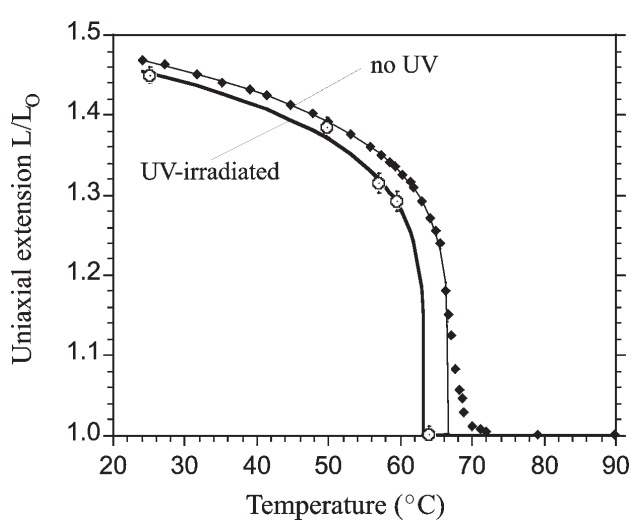

FIG. 11: The asymptotic values of $\lambda(t, T)$, for Azo18 irradiated for a long time at several fixed temperature, after correcting for the temperature increase associated with irradiation, $\beta\left[n_{0}-n\right]$, which correspond well to the idea of a shifted critical temperature $T^{*}$, as illustrated theoretically in Fig. 6

cal temperature of the nematic-isotropic phase transition by an amount

$$
\Delta T=\beta\left[n_{0}-n_{\infty}(T)\right]=\beta n_{0} \frac{\tau_{c t} \eta}{1+\tau_{c t} \eta},
$$

which is dependent on the intensity of the ultraviolet light used (through $\eta$ ). Hence, a slightly modified form of $\lambda=1+\alpha Q$, making only the replacement $T_{\mathrm{ni}} \rightarrow$ $T^{*}$, should fully describe the final, steady-state length of the sample, $\lambda_{\mathrm{uv}}(t \rightarrow \infty)$, as a function of temperature. All the other parameters in Eq. (5), in particular the exponent $\xi$, should remain unchanged. Indeed, one finds that this idea does describe $\lambda_{\mathrm{uv}}(t \rightarrow \infty)$ very well, as shown in Fig. 11. The corresponding value of shift $\Delta T$, which best fits the data in Fig. 11, is found to be $(3.3 \pm$ $0.7)^{\circ} \mathrm{C}$ which agrees well with the average asymptotic value of $\beta\left[n_{0}-n_{\infty}\right]$ gleaned from extrapolating the data sets in Figs. 778, of $(3.5 \pm 1.9)^{\circ} \mathrm{C}$.

This work, has studied a wide range of photosensitive materials, based on azobenzene molecular moieties, whose differing compositions and topologies are reflected in their varied mechanical response to irradi- 
ation with ultraviolet light. The fundamental details of this response were investigated, in particular its kinetics and the variation of the critical temperature near the nematic-isotropic phase transition. The obtained experimental results are very well described by a theoretical model assuming a simple linear shift in the critical temperature with the concentration of kinked cis groups, which results in a rather non-trivial model for the dependence of the underlying nematic order parameter $Q$ and macroscopic uniaxial mechanical extension $\lambda$ on irradiation time and temperature. Basic material parameters and constants were also determined, which will allow subsequent investigations to further probe the details of this fascinating phenomenon. With such a novel, non-invasive method of control over mechanical actuation, there is a great potential for application, but still very much is left unknown.

\section{Acknowledgments}

We are grateful to Wacker Chemie for donating the platinum catalysts for the chemical preparations, and to H. Finkelmann and M. Warner for a number of useful discussions and advice. This work has been supported by EPSRC UK.
[1] W. Gleim and H. Finkelmann, in: Side-Chain Liquid Crystal Polymers, Ed. C.B. McArdle (Blackie \& Sons, 1989), p. 287.

[2] G. G. Barclay and C. K. Ober, Progr. Polym. Sci., 18, 899 (1993).

[3] H. R. Brand and H. Finkelmann, in: Handbook of Liquid Crystals, Ed D. Demus et al. (Wiley-VCH, Weinheim, 1998), Vol.3, Chapter V.

[4] E. M. Terentjev, J. Phys. Cond. Mat., 11, R239 (1999).

[5] M. Warner and E. M. Terentjev, Prog. Polym. Sci., 21, 853 (1996).

[6] J. Küpfer and H. Finkelmann, Macromol. Rapid Comm. 12, 717 (1991).

[7] N. Assfalg and H. Finkelmann, Kaut. Gummi. Kunstst. 52, 677 (1999).

[8] H. Wermter and H. Finkelmann, e-Polymers (www.epolymers.org) no.013 (2001).

[9] S. M. Clarke, A. Hotta, A. R. Tajbakhsh, and E. M. Terentjev, Phys. Rev. E 64, 061702 (2001).

[10] A. R. Tajbakhsh and E. M. Terentjev, Euro. Phys. J. E 6, 181 (2001)..

[11] J. E. Huber, N. A. Fleck and M. F. Ashby, Proc. R. Soc. Lond. A, 453, 2185 (1997).

[12] M. Hebert, R. Kant and P.-G. de Gennes, J. Phys. I France, 7, 909 (1997).

[13] D. L. Thomsen III, P. Keller, J. Naciri, R. Pink, H. Jeon, D. Shenoy and B. R. Ratna, Macromolecules, 34, 5868 (2001).

[14] C.-C. Chang, L.-C. Chien and R. B. Meyer, Phys. Rev. $E, \mathbf{5 6}, 595$ (1997).

[15] H. Finkelmann, E. Nishikawa, G. G. Pereira and M. Warner, Phys. Rev. Lett., 87, 015501 (2001).

[16] S. Tazuke, S. Kurihara and T. Ikeda, Chem. Lett., (1987)
911.

[17] T. Ikeda, S. Horiuchi, D.B. Karanjit, S. Kurihara and S. Tazuke, Macromolecules, 23, 36 (1990); / T. Ikeda and O. Tsutsumi, Science, 268, 1873 (1995); / O. Tsutsumi, Y. Demachi, A. Kanazawa, T. Shiono, T. Ikeda and Y. Nagase, J. Phys. Chem. B, 102, 2869 (1998).

[18] C. H. Legge and G. R. Mitchell, J. Phys. D: Appl. Phys., 25, 492 (1992).

[19] I. Janossy and L. Szabados, J. Nonlin. Opt. Phys. Mater., 7, 539 (1998).

[20] D. Y. Kim, S. K. Tripathy, L. Ki, J. Kumar, Appl. Phys. Lett., 66, 1166 (1995).

[21] I. Sample, New Scientist, 2302 (2001) 18; /

F. Harvey, Financial Times, 10-Sep-2001.

[22] H. Finkelmann, A. Greve and M. Warner, Euro. Phys. J. $E, \mathbf{5}, 281$ (2001).

[23] N. Boden, R. J. Bushby and L. D. Clark, J. Chem. Soc. Perkin Trans. I, (1983) 543.

[24] Vogel, Textbook of Practicle Organic Chemistry (Wiley \& Sons), 5th Edition, p. 957.

[25] S. K. Prasad and G. G. Nair, Adv. Mater., 13, 40 (2001); / P. Vanoppen, P. C. M. Grim, M. Rcker, S. De Feyter, G. Mössner, S. Valiyaveettil, K. Müllen and F. C. De Schryver, J. Phys. Chem., 100, 19636 (1996).

[26] P. M. Chaikin and T. C. Lubensky, Principles of Condensed Matter Physics, (CUP, Cambridge 1995).

[27] J. Cardy, Physica A, 263, 215 (1999).

[28] F. Elias, S. M. Clarke, R. Peck and E. M. Terentjev, Europhys. Lett., 47, 442 (1999).

[29] P.-G. de Gennes and J. Prost, Physics of Liquid Crystals (Clarendon Press, Oxford, 1993). 